\title{
The Case of "Rosa": The Importance of Specificity in Our Quest to Integrate Cultural Competence in Practice
}

\author{
YAMILKA URQUIZA MENDOZA ${ }^{\text {a,b }}$ \\ a Alliant International University, Graduate Department of Marriage and Family Therapy, San Diego, CA. \\ ${ }^{\mathrm{b}}$ Correspondence regarding this article should be sent to Yamilka Urquiza Mendoza, Graduate Department of \\ Marriage and Family Therapy, Alliant International University, 10455 Pomerado Road, San Diego, CA 92131. \\ Email: yurquiza@alliant.edu
}

\begin{abstract}
This commentary explores Nicole Vigoda Gonzalez's (2018) treatment of "Rosa" with Accelerated Experiential Dynamic Psychotherapy (AEDP), an attachment-based, relational, and experiential approach effective for treating attachment trauma. I discuss how AEDP also welcomes culturally competent strategies. My focus is the importance of specificity in the inclusion of culturally competent strategies in psychotherapy practice. Specifically, I address the difference between a Caribbean versus an Hispanic identification. I also explore the impact of culture, including race/ethnicity and familial discrimination, in Rosa's case. Finally, I explore the impact of the therapist's disclosure, or lack thereof, of her own race/ethnicity in the context of AEDP interventions.
\end{abstract}

Key Words: cultural competence; culture; specificity; race; ethnicity; discrimination; familial discrimination; disclosure; Hispanic; Caribbean; Cuban; language; attachment trauma; change; emotional experience; Accelerated Experiential Dynamic Therapy (AEDP); case study; clinical case study

\section{INTRODUCTION}

Cultural competence is a term buzzing in the ears of therapists and of those engaged in the development and/or improvement of therapeutic modalities and theories. In the case of Rosa, Nicole Vigoda Gonzalez (2018) explores the integration of culturally competent strategies into the trauma treatment of her client, Rosa, utilizing Accelerated Experiential Dynamic Psychotherapy (AEDP) interventions. AEDP welcomes with humility the ongoing process of learning about people of diverse backgrounds by honoring, acknowledging, and accepting the differences.

I enjoyed Vigoda Gonzalez's exploration of therapeutic modalities, culminating with her arriving at AEDP as the modality of choice, with interventions that best suited Rosa. This makes sound clinical sense as a treatment of choice for someone with relational trauma, like Rosa. The AEDP therapist has, as a rule, a warm, welcoming, and holding stance; and AEDP interventions focus on relational, attachment-based, and emotion-processing principles (Fosha, 2000). The AEDP therapist is affirming, emotionally engaged, explicitly empathic, and seeks to be a "True Other" as she/he responds to a client's needs (Fosha, 2005). Thus, the AEDP approach appeared 
perfectly suited for working through Rosa's trauma, and also for welcoming her ethnicity and native language.

This paper is a commentary on Vigoda Gonzalez's case study, including the use of AEDP in her work, and on this therapy viewed through a cultural lens. I explore issues that include the difference between the terms Caribbean versus Hispanic; race/ethnicity and discrimination; and self-disclosure, or lack thereof, of language/race/ethnicity in the context of AEDP interventions. I also comment on AEDP and first/second language in therapy, and AEDP interventions to heal attachment trauma. My commentary includes a brief self-disclosure of my own cultural and language background.

\section{AEDP IN ACTION IN THE CASE OF ROSA}

As we experience from the case report, Vigoda Gonzalez is an attuned therapist who closely tracked Rosa's experiences. First, she tracked Rosa's responses to different therapeutic modalities, including a confrontational short-term dynamic psychotherapy approach to which "Rosa appeared to become affectively flooded and more guarded" (p. 14). Vigoda Gonzalez then went on to try the non-confrontational approach of AEDP to which "Rosa was significantly more receptive" (p. 14). Noticing the impact chosen treatment modalities had on the Rosa's experience and openness, Vigoda Gonzalez wisely stayed with an attachment-based, relational, and experiential model like AEDP. Putting her excellent attunement skills in action, Vigoda Gonzalez made good use of central AEDP techniques, including relational, emotion processing, and metaprocessing interventions.

As we learn from Vigoda Gonzalez's report, Rosa had experienced a lifetime of having no reliable, accessible attachment figures to count on. The rejection and lack of responsiveness she experienced from her mother were inherently traumatizing and contributed to her developing an insecure style of attachment. Working within the principles and spirit of the AEDP model and utilizing specific AEDP interventions, the therapist was able to help Rosa transform that insecure attachment style into a secure one, thus enhancing Rosa's ability to regulate and express affect, process information, and communicate authentically in the presence of a therapist she clearly experienced as a "True Other" (Fosha, 2005).

\section{THERAPY THROUGH A CULTURAL LENS}

Reviewing the literature on the subject, Vigoda Gonzalez addresses the importance of race/ethnicity-matching in psychotherapeutic treatment. Though she makes mention of Rosa's Caribbean background, nevertheless later on, she identifies Rosa as Hispanic. This presents us with some challenges and points to some gaps.

In light of that, I would like to develop three themes: (a) the difference between a Caribbean versus an Hispanic identification; (b) the impact of culture, including race/ethnicity and familial discrimination in Rosa's case; and (c) an exploration of the impact of the therapist's disclosure, or lack thereof, of her own native language/race/ethnicity in the context of AEDP interventions. 


\section{EXPANDING ON THE DIFFERENCE OF CARIBBEAN VERSUS HISPANIC IDENTITIES: DOES ROSA IDENTIFY AS CARIBBEAN OR HISPANIC? THE DISTINCTION IS IMPORTANT}

The term "Hispanic" is used in the United States to group together people of Cuban, Mexican, Puerto Rican, South or Central American, or other Spanish culture of origin regardless of race (Census Bureau, rev. 2018). Furthermore, people identified as Hispanic can also be U.S. born, with parents or ancestors who migrated to the United States from different countries, cultures, and very different backgrounds. The use of the term "Hispanic" can lead people to assume that all people who speak Spanish, i.e., "Hispanics," share equal cultural heritage, which they do not. Relying on the overly general term "Hispanic" risks disregarding the specificity of people's cultural experiences.

Therapists should optimally make decisions about how to incorporate cultural elements tailored to the person's unique racial/ethnic background, and not limit themselves to just honoring the client's linguistic background. Vigoda Gonzalez said that Rosa was a "Hispanic woman" (p. 2), later on adding that Rosa was "born in a Spanish Caribbean island" (p. 13). Although Spanish speaking, Spanish-Caribbean people have their own culture and heritage, quite different and distinct from what it is commonly known as "Hispanic" in the United States. This is important to mention in the case of Rosa, as, for the most part, Spanish-Caribbean race and racial identity are viewed differently among Spanish speaking people than they are in the United States where, for some, "one's culture is one's race," (discussed above, in this issue, in Rodriguez, 2018).

There are 26 Caribbean countries including territories of other nations (Travel Republic, 2016), and three of them speak Spanish-Caribbean. The Caribbean islands are each unique in their cultural background, reflecting many influences: the influx of immigrants, voluntary or forced, from Spanish, Portuguese, Dutch, French, African backgrounds; slavery; laborers; colonization; and decolonization (Lipski, 1993). Some of the Islands are financially more stable than others.

Rosa and her family's cultural identity would most likely have been influenced by her/their Caribbean Island's culture, including language, race, religion, and socioeconomic status of the family.

\section{EXPLORING THE IMPACT OF ROSA'S CULTURE INCLUDING RACE/ETHNICITY AND FAMILIAL DISCRIMINATION}

\section{Culture}

Culture functions as a way to maintain, adjust, and achieve the well-being of the individual, family, and community (Friedemann, 1995), by guiding how to best utilize the available resources within the family and community (Friedemann, Buckwalter, Newman, \& Mauro, 2013). For some, this means utilizing those resources for the benefit of the individual, and for others it means doing so for the benefit of the family and community (Pinquart $\&$ 
Sörensen, 2005). From Vigoda Gonzalez's report, it appears that Rosa was seeking to achieve the wellbeing of her family. In light of that, some questions that are important to address come to mind: Where does Rosa locate herself culturally? In light of her cultural orientation, where does she want to locate her resources, given her initial concerns to help her son?

The following example raised some red flags for me. In response to the therapist's initial focus on her early traumatic experiences in the family, Rosa says: "I can't look at this right now, it is too much to deal with... I need to focus on my son" (p. 14). In response to this, Vigoda Gonzalez writes: "I hypothesized that focusing on her son's drinking functioned as defensive avoidance of the overwhelming feelings triggered by those traumatic experiences." (p. 14). Later Vigoda Gonzalez writes: "As I pointed to her efforts to stay away from those topics and their associated emotions, she capitalized on her desire to focus on her son" (p. 21).

For Rosa, family was extremely important. Continuing to treat Rosa's own goal of treatment as primarily a defense against Rosa focusing on her own experiences and feelings appears to obscure the culturally congruent importance Rosa places on taking care of her son. Furthermore, given Rosa's history of feeling alienated, there is a risk she may feel even less seen or understood with a therapist who viewed her culturally appropriate goal as a defense mechanism. Not honoring her stated goal of therapy runs the risk of being culturally insensitive and of pathologizing Rosa's culturally congruent commitment to her son.

\section{Race/Ethnicity and Discrimination}

Vigoda Gonzalez mentions Rosa was "frequently criticized and humiliated by her mother due to her darker skin tone and coarser hair" (p. 13). Racial discrimination is emotionally painful (Carter, 2007) and in this case, appears to be a major factor contributing to Rosa's family-oforigin trauma. Rosa would have benefited from the therapist making space for and exploring with Rosa how Rosa's being alienated by her own family and socially discriminated for her skin color affected her view of herself and others in a society with people of diverse backgrounds.

Also potentially fruitful would have been to explore how Rosa and her family were influenced by migration to the United States. Did they view race/ethnicity differently as they tried to assimilate to a new culture? In the transcript, the therapist mentions providing "psychoeducation about racial micro-aggressions" (p. 30) and other forms of discrimination. Yet it is not clear whether AEDP interventions were utilized to help Rosa through the pain and anger she may have felt as a result of the racial discrimination she suffered, including in her own family.

\section{THE ROLE OF THE THERAPIST'S DISCLOSURE, OR LACK THEREOF, OF HER OWN LANGUAGE, RACE/ETHNICITY IN THERAPY IN GENERAL, AND WITH AEDP INTERVENTIONS IN PARTICULAR}

It can be said that language is full of flavor, emotion, and history. This is evident in how the Spanish-Caribbean islands have been influenced by the languages and dialects brought to the islands through settlement and colonization, including Spanish, Portuguese, Dutch, and Yoruba 
(Lipski, 1993). This is relevant here because Rosa's language is colored through the richness of culture. In fact, Kiderman, Dressler, and Freedman-Stewart (2011) reported the possibility of misunderstanding in treatment with patients of shared mother tongue, if one fails to interpret history in the patients' own cultural terms.

Although she waited until sometime between sessions 3 and 9, I love that Vigoda Gonzalez noticed and seized the moment (see Frederick, quoted in Prenn \& Fosha, 2016) and self-disclosed her bilingualism. This aligns well with AEDP's encouragement for the therapist to judiciously self-disclose (Prenn, 2009), always in the service of the patient. This was one of many actions taken by Vigoda Gonzalez in an effort to provide as safe a place as she could for Rosa.

Vigoda Gonzalez's own race or ethnic background was not shared in the case report. We only know that Vigoda Gonzalez has the same first language as Rosa. The case study, and possibly the treatment, would have benefited from a clear picture of how and where Vigoda Gonzalez locates herself racially and ethnically in comparison to Rosa, and how this did or did not impact the treatment process utilizing AEDP.

The treatment might have also become further enriched through the explicit exploration of both similarities and differences between Rosa and Vigoda Gonzalez's Spanish background, both linguistic and cultural. How much was Rosa's perception of her therapist's accessibility and responsiveness the result of her race/ethnicity and how much the result of her AEDP interventions? Lacking that information, we are not in a position to answer that question.

In my practice, the vast majority of my clients have been of Spanish-speaking origin. As part of the relationship building, I usually disclose my origins as well as my bilingualism. Disclosure usually tends to elicit curiosity in clients. There is often a shift in their affect from an apparently high level of anxiety about therapy to a calmer state. Many clients choose to do the therapy using their first language. Other clients chose to continue using their second language throughout treatment and only at times shift to their native tongue, if needed.

I wish to note an important observation based on my own clinical experience. In the treatment of bilingual clients who choose to do the therapy utilizing their second language, AEDP interventions have consistently helped my clients, just as when we are speaking Spanish. With AEDP, we have been able to work with emotionally charged experiences and process them through to completion. This makes clear that speaking one's second (or third or more) language is not an automatic barrier to deep trauma processing and resolution.

\section{AEDP AND LANGUAGE}

In AEDP, giving ample space for recalling experiences by the client in their native language is congruent with the ethos of the model. The AEDP therapist as a rule normalizes and validates the client's experience and shows appreciation for any attempt on the part of the client to deepen contact with experience. An AEDP therapist may say, "I hear that this memory you are trying to translate is so important for you to share with me. I am so appreciative of you 
sharing this with me." Given that the AEDP therapist looks for non-verbal resonance, in my experience by tracking present moment-to-moment affect, defenses can often be bypassed without the need to use or find specific language content.

We learn from Vigoda Gonzalez that "when Rosa translated affectively-charged experiences from the primary language in which they occurred, from Spanish into English, her narrative seemed to lose emotional vibrancy, and she appeared detached from those experiences" (p. 18, italics added). Although speaking a second language can indeed at times create emotional distancing, it is important to state that relying on a second language is not always a defense. In my own experience, personally and in my own therapeutic work, I have clearly struggled attempting to translate a word. The very effort to do so took me away from an emotionally charged experience I had no intention of disconnecting from.

This is important, given that some of the trauma processed in treatment happened during childhood, when it is assumed Rosa mainly spoke Spanish. However, other traumas for Rosa occurred throughout the different stages of her adult life. During Rosa's adult life, it is not clear if the language involved in the traumatic experiences was her native language of Spanish, her second language of English, or maybe both. Memories are said to be more intense in the language in which they are registered (Harris, Berko Gleason, \& Ayçiçegi, 2006; see also Vigoda Gonzalez, 2918). Surprise and excitementugh not mentioned by Vigoda Gonzalez, the same traumatic memories could have been recoded from her native to the second language (Schrauf \& Durazo-Arvizu, 2016), given her age at migration and or her tendency to switch between languages.

Speaking in a person's second language does not always create, or need not create, emotional distance, and if it does, that distance can be addressed in a variety of ways in AEDP therapy, in addition to language switching. There are several factors contributing to this. For example, translation, in which case AEDP's interventions could work through by setting aside the need to translate (in case of a non-bilingual therapist) and honoring the client's present moment stance, and by being a secure base for the client (e.g., through unconditional acceptance of the client). "I see that you are trying to translate, could you just say it? No need to translate." As the client speaks, the AEDP therapist is tracking moment-to-moment nonverbal aspects of the experience. The intervention facilitates continued co-creation of a safe therapeutic relationship. It also creates opportunities for slowing down and expansion of experiences through dyadic work to shift client's internal working model by disconfirming echoes of helpless aloneness in the world.

A shared language between therapist and client, regardless of whether it is the native language or not, does have some benefits, as Vigoda Gonzalez's case of Rosa demonstrates. In my own experience with bilingual speaking clients, language switching in sessions facilitates the therapeutic process. There is a sense of flow when the client and therapist share a language and/or when the client has the perception of being understood in that language by therapist. The client's ability to express common phrases and or memories in the moment, without the need to translate, enhances the sense of feeling understood and seen, and increases feelings of safety and connectedness. Similarities can bring delight to the therapeutic relationship. Rosa, "reacted 
with surprise and excitement" (p. 14) to Vigoda Gonzalez's self-disclosure of the commonality between the two of them.

As we learn from Vigoda Gonzalez, a "judicious self-disclosure of [her] own bilingualism was deemed clinically appropriate" (p. 18). The disclosure appeared to delight Rosa “'I'm so very happy you speak Spanish"” (p. 25). However, since the case study highlighted the importance of integrating the client's native language, identifying that factor as a pivotal part of the treatment, more could have been done with it. In addition to supporting Rosa to use Spanish especially when dealing with traumatic experiences from early childhood, the treatment, and especially the trauma work, might have been further enhanced if the therapist worked with the client to slow down, and to explore Rosa's fluency in her native tongue.

In my work with Spanish speaking clients from different countries, with different levels of acculturation and of fluency in their first and second languages, AEDP continues to bring healing. Central to its being an experiential treatment, AEDP actually aims to drop below the level of language to the level of bodily-based emotion and connection. AEDP invites the person as a whole to the therapy room.

Again in my experience, AEDP supports embodying a warm, personable, welcoming stance that people from the various Spanish-speaking countries enjoy and find attractive in a therapeutic relationship. I always honor the client's request to use either Spanish or English, or both. Finally, it has been my experience that AEDP interventions are very effective in bypassing challenges, including language and ethnic background, and actually turning them into relational strengths.

\section{AEDP AND WORK TO HEAL ATTACHMENT TRAUMA}

Rosa's specific goals, which developed during the treatment, to address her attachment trauma were achieved with AEDP. The author helped Rosa undo her experience of aloneness as a child, where she had been bullied by her siblings, and where she had a mother who was neither emotionally accessible nor engaged. Following AEDP principles and her own intuition and attunement, the therapist was able to co-create with Rosa a safe environment where her client was able to do the work that allowed her to come to a very different understanding of the self and others. According to Bowlby (1973), internal working models are formed, elaborated, maintained, and changed through emotional experience. Rosa's internal working model, developed in childhood in an unsafe environment, was transformed. In a safe setting, Rosa's emotions were processed and her family of origin trauma was deeply seen, recognized, and honored by her therapist practicing in a manner informed by AEDP.

AEDP not only works with trauma, it has a healing orientation that focuses on transformation as both a goal and experience (Fosha, 2009). The positive affective phenomena referred to by Fosha (2009) include affective experiences, even those that are not so pleasant or easy, e.g., grief, but feeling true to the client, including naturalness, energy, clarity, effectiveness, contact and relatedness, and extreme positive affects. According to Fosha (2009), the healing power of AEDP transforms suffering and gives rise to transformational affects and core state, by 
organically linking opposing realms of suffering and flourishing. This is done through "the methodologies of dyadic affect regulation, experiential processing of experience to completion, and the metaprocessing of transformational experience" (Fosha, 2009, p. 260). In the case of Rosa, there was clear delight as Rosa expressed to Vigoda Gonzalez her gladness of the shared language. Within the context of AEDP therapy, an important option for Vigoda Gonzales would have been to stay with this experience of gladness in the dyad, processing it somatically through to completion, and then metaprocessing the experience for Rosa cognitively, affectively, and relationally.

Rosa's flourishing transformational affects were evident with her expression of deeply positive feelings about being in this therapy and its positive impact on her life: e.g., "'I am so glad I'm coming here," "I feel invigorated as I'm getting ready to come here," and "This has been so helpful... It's like I'm finally seeing things clearly" (p. 34). The therapist might have stayed with Rosa's experience of "finally seeing things clearly;" had she done that, it might have been possible to further deepen the further transformational affects to facilitate the even greater expansion of Rosa's new experience.

\section{CONCLUSION}

In reviewing Vigoda Gonzalez's accounts of her work with Rosa, I was reminded of the importance of specificity in the inclusion of cultural strategies in practice. It is clearly useful for major organizations like the United States Census to gather information by grouping people in broad categories, i.e., by regions or languages. However, in a psychotherapy practice that focuses on the uniqueness of the individual's experience, this is not the case: even with the intention of honoring multicultural practices, the use of overly broad categories risks ignoring each client's cultural being, along with years of history that impact the concerns brought to treatment.

Also, let us not forget the impact language can have in treatment. Linked to a person's culture, language provides a powerful window to hearts ready to heal. Held by the symbolic hand of a warm and welcoming AEDP therapist who is experienced as a "True Other," clients can heal relational trauma.

Finally, as is vividly illustrated in the case of Rosa, AEDP's attachment-based and emotion-processing-oriented principles (Fosha, 2000) welcome and are affirmatively responsive to diverse cultural backgrounds.

\section{REFERENCES}

Bowlby, J. (1973). Attachment and loss. Volume II: Separation, anxiety and anger. London: Hogarth Press.

Carter, R. T. (2007). Racism and psychological and emotional injury: Recognizing and assessing race-based traumatic stress. Counseling Psychologist, 35(1), 13-105.

Census Bureau (rev. 2018). Hispanic origin. Retrieved from https://www.census.gov/topics/population/hispanic-origin/about.html 
Fosha, D. (2000). The transforming power of affect: A model for accelerated change. New York, NY: Basic Books.

Fosha, D. (2005). Emotion, true self, true other, core state: Toward a clinical theory of affective change process. Psychoanalytic Review, 92(4), 513-552.

Fosha, D. (2009). Positive affects and the transformation of suffering into flourishing. Annals of the New York Academy of Sciences, 1172, 252-262.

Friedemann, M. L. (1995). The framework of systemic organization: A conceptual approach to families and nursing. Thousand Oaks, CA: Sage Publications.

Friedemann, M., Buckwalter, K., Newman, F., \& Mauro, A. (2013). Patterns of caregiving of Cuban, other Hispanic, Caribbean black, and white elders in South Florida. Journal of Cross-Cultural Gerontology, 28(2), 137-152.

Harris, C.L., Berko Gleason, J. \& Aycicegi, A. (2006) .When is a first language more emotional? Psychophysiological evidence from bilingual speakers. In A. Pavlenko (Ed.), Bilingual minds: Emotional experience, expression and presentation (pp. 257-283). Tonawanda, N.Y.: Multilingual Matters.

Kiderman, A., Dressler, R., \& Freedman-Stewart, B. (2011). Are we speaking the same language? Journal of Evaluation in Clinical Practice, 17(2), 328-329. doi:10.1111/j.13652753.2010.01438.x

Lipski, J.M. (1993). On the non-creole basis for Afro-Caribbean Spanish. Retrieved from the Digital Repository of the University of Mexico: http://digitalrepository.unm.edu/laii research/18

Pinquart, M., \& Sörensen, S. (2005). Ethnic differences in stressors, resources, and psychological outcomes of family caregiving: A meta-analysis. The Gerontologist, 45(1), 90-106. doi:10.1093/geront/45.1.90

Prenn, N. (2009). I second that emotion! On self-disclosure and its metaprocessing. In A. Bloomgarden, \& R. B. Menutti, (Eds.), The therapist revealed: Therapists speak about self-disclosure in psychotherapy (pp. 85-99). New York, NY: Routledge.

Prenn, N. \& Fosha, D. (2016). Supervision essentials for Accelerated Experiential Dynamic Psychotherapy. Washington DC: APA Press.

Rodriguez, R. (2018). The case of "Rosa": Exploring socio-cultural identities in treatment. Pragmatic Case Studies in Psychotherapy, 14(2), Article 3, 131-137. Available: http://pcsp.libraries.rutgers.edu

Schrauf, R. W., \& Durazo-Arvizu, R. (2016). Bilingual autobiographical memory and emotion: Theory and methods. In Pavlenko, A. (Ed.), Bilingual minds: Emotional experience, expression and presentation (pp. 284-311). Tonawanda, N.Y.: Multilingual Matters.

Travel Republic Limited (2016). How many of the 26 Caribbean countries have you heard of? Retrieved from https://www.travelrepublic.co.uk/blog/26-countries-of-the-caribbean

Vigoda Gonzales, N. (2018). The merits of integrating accelerated experiential dynamic psychotherapy and cultural competence strategies in the treatment of relational trauma: The case of "Rosa. Pragmatic Case Studies in Psychotherapy, 14(1), Article 1, 1-57. Available: http://pcsp.libraries.rutgers.edu. http://dx.doi.org/10.14713/pcsp.v14i1.2032 